\title{
Deformations Monitoring by Integrating Local and Global Reference Systems
}

\author{
J. Zurutuza \\ GEOLan Donosti SL, Portuetxe 53 b Of. 314, 20.018 San Sebastián (Gipuzkoa). \\ jz@geolandonosti.com \\ M. J. Sevilla \\ Instituto de Astronomía y Geodesia (CSIC-UCM), \\ Facultad de Ciencias Matemáticas, Universidad Complutense de Madrid, 28040 Madrid, Spain. \\ sevilla@mat.ucm.es
}

\begin{abstract}
Although the transformation between threedimensional reference systems has been widely studied in geodesy, in this paper, a different way of integrating, and not transforming, both systems is used. On one hand, a straightforward computational method without iterations nor "a priori" known values is shown. On the other hand, some applications have been made and presented. The method, recently recognised by Grafarend, is known as the Procrustes solution and it is based on the Singular Value Decomposition of a matrix from which we obtain the Euler Elements. Finally real network located in Gipuzkoa has been used to monitor a simulated deformation via the Procrustes solution, which is compared with the Helmet's datum transformation classical approach.
\end{abstract}

Keywords: Procrustes, SVD, datum transformation, Euler elements, Orthogonal transformation.

\section{Introduction}

Still today, one of the most challenging tasks in geodesy is the datum transformation. We can admit that the datum transformation problem has never been as important as nowadays that a new global reference system has been adopted: ETRS89. Thus, obtaining correct and accurate transformation models from one system to other (global to global, global to local or local to local) is a main task in modern geodesy to warrant that there is no loose of accuracy in the data due to the transformation. Although the datum transformation has been widely studied by many authors (Krakiwsky and Thompson, 1974), this paper deals with the method known as the Procrustes problem (Schönemann, 1966, Sansò, 1973, Crosilla, 1983, Awange, 2003, Awange and Grafarend, 2005). The basic aim of the
Procrustes solution is to fit one given set of points into another known set of points and get a measure of the quality. In the next pages, the different solutions will be treated in detail and an example is provided were transformation models are compared, using real data.

\section{Three Dimensional Positioning Systems}

In order to understand the Procrustes solution, the local and the global three dimensional positioning systems must be defined. The main characteristics of such reference systems are pointed in the next lines.

\subsection{Local Positioning System}

The local positioning system (Figure 1) owes its name to the following characteristic: every measure is referred to a local origin which is, usually, the centre of the instrument. The local positioning system $\left(f_{1}, f_{2}, f_{3}\right)$ can be described as follows:

- Origin: Centre of the instrument.

- $\mathrm{f}_{3}$ is the direction of the plumb line.

- $\mathrm{f}_{1}$ in the horizontal plane.

- $\mathrm{f}_{2}$ form a right handed system with $\mathrm{f}_{3}$ and $\mathrm{f}_{1}$

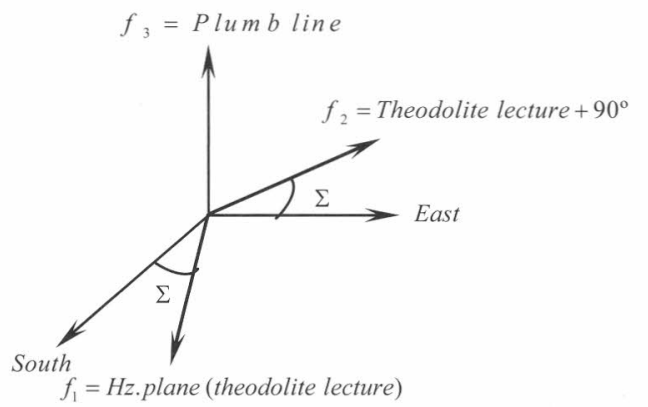

Fig. 1: Local positioning system. 


\subsection{Global Positioning System}

The main characteristics of the global positioning system $\left(F_{1}, F_{2}, F_{3}\right)$ are (Figure 2$)$ :

- Origin: Center of mass of the Earth.

- $\mathrm{F}_{3}$ is the ITRS pole direction.

- $\mathrm{F}_{1}$ is the ITRS Origin of longitudes.

- $\mathrm{F}_{2}$ forms aright handed system with $\mathrm{F}_{1}$ and $\mathrm{F}_{3}$.

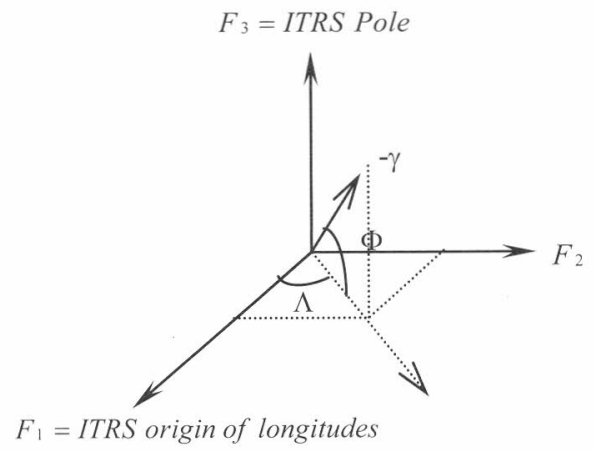

Fig. 2: Global positioning system.

\section{Three Dimensional Datum Transformation Models}

\subsection{The Helmert Transformation}

The classical Helmert datum transformation solution is given by a translation (vector $\mathbf{T}$ ), a scale factor $k$ and a rotation (matrix $\mathbf{R}(\omega)$ ). The most important disadvantages of Helmert's solution are: they need "a priori" known values, they are iterative and need to be linearized. Commonly, the solution is presented by the equations

$$
\left[\begin{array}{c}
X \\
Y \\
Z
\end{array}\right]_{G P S}=\left[\begin{array}{l}
T_{U} \\
T_{V} \\
T_{W}
\end{array}\right]_{\text {Trastation }}+(1+k)\left[\begin{array}{ccc}
1 & \omega_{W} & -\omega_{V} \\
-\omega_{W} & 1 & -\omega_{U} \\
\omega_{V} & \omega_{U} & 1
\end{array}\right]\left[\begin{array}{c}
U \\
V \\
W
\end{array}\right]_{\text {Local }}(1)
$$

The parameters of the transformations are obtained by the least squares mixed conditional model

$$
\mathbf{A x}+\mathbf{B v}-\mathbf{t}=\mathbf{0},
$$

were $\mathbf{A}$ and $\mathbf{B}$ are the design matrices, $\mathbf{x}$ the parameter vector, $\mathbf{t}$ the vector of observations and $\mathbf{v}$ the residuals.

\subsection{The Procrustes Transformations}

The weighted extended orthogonal Procrustes (WEOP) transformation in given by the equation (Awange and Grafarend 2005)

$$
\mathbf{x}=\mathbf{u} \mathbf{T}^{\mathrm{T}}+k \mathbf{X} \mathbf{R}^{\mathrm{T}}+\mathbf{E}
$$

were $\mathbf{E}$ represents the residuals, $\mathbf{x}, \mathbf{X}$, the coordinates of several points in each system respectively, $k$ the scale factor, $\mathbf{R}$ the rotation matrix and $\mathbf{u}$ is a vector of as many ones as known points in both systems. $\mathbf{T}$ is the translation vector.

To see how (3) is obtained we need to know how a transformation of a point is performed. To transform the coordinates of a point from a known reference system into another known reference system a translation vector, a rotation matrix and a scale factor shall be considered. Therefore, for one single point we have:

$$
\left(\begin{array}{l}
x \\
y \\
y
\end{array}\right)=\left(\begin{array}{l}
T_{1} \\
T_{2} \\
T_{3}
\end{array}\right)+k\left(\begin{array}{lll}
r_{11} & r_{12} & r_{13} \\
r_{21} & r_{22} & r_{23} \\
r_{31} & r_{32} & r_{33}
\end{array}\right)\left(\begin{array}{c}
X \\
Y \\
Z
\end{array}\right)
$$

In case of two points, (4) would be:

$$
\left(\begin{array}{ll}
x_{1} & x_{2} \\
y_{1} & y_{2} \\
z_{1} & z_{2}
\end{array}\right)=\left(\begin{array}{ll}
T_{1} & T_{1} \\
T_{2} & T_{2} \\
T_{3} & T_{3}
\end{array}\right)+k\left(\begin{array}{lll}
r_{11} & r_{12} & r_{13} \\
r_{21} & r_{22} & r_{23} \\
r_{31} & r_{32} & r_{33}
\end{array}\right)\left(\begin{array}{cc}
X_{1} & X_{2} \\
Y_{1} & Y_{2} \\
Z_{1} & Z_{2}
\end{array}\right)
$$

For n points, (4) would be written as:

$$
\begin{aligned}
\left(\begin{array}{llll}
x_{1} & x_{2} & \ldots & x_{n} \\
y_{1} & y_{2} & \ldots & y_{n} \\
z_{1} & z_{2} & \ldots & z_{n}
\end{array}\right) & =\left(\begin{array}{llll}
T_{1} & T_{1} & \ldots & T_{1} \\
T_{2} & T_{2} & \ldots & T_{2} \\
T_{3} & T_{3} & \ldots & T_{3}
\end{array}\right)+ \\
& +k\left(\begin{array}{llll}
r_{11} & r_{12} & r_{13} \\
r_{21} & r_{22} & r_{23} \\
r_{31} & r_{32} & r_{33}
\end{array}\right)\left(\begin{array}{cccc}
X_{1} & X_{2} & \ldots & X_{n} \\
Y_{1} & Y_{2} & \ldots & Y_{n} \\
Z_{1} & Z_{2} & \ldots & Z_{n}
\end{array}\right)
\end{aligned}
$$

We consider the matrices of the coordinates:

$$
\mathbf{x}=\left(\begin{array}{ccc}
x_{1} & y_{1} & z_{1} \\
x_{2} & y_{2} & z_{2} \\
\cdots & \cdots & \cdots \\
x_{n} & y_{n} & z_{n}
\end{array}\right) \text { and } \mathbf{X}=\left(\begin{array}{ccc}
X_{1} & Y_{1} & Z_{1} \\
X_{2} & Y_{2} & Z_{2} \\
\cdots & \cdots & \cdots \\
X_{n} & Y_{n} & Z_{n}
\end{array}\right)
$$

Taking into account the notation used in (3), and the Cartesian coordinates in (7), the transformation equation for $n$ points can be rewritten as:

$$
\mathbf{x}^{\mathrm{T}}=\mathbf{T} \mathbf{u}^{\mathrm{T}}+k \mathbf{R} \mathbf{X}^{\mathrm{T}}
$$


By transposing (8) we get:

$$
\mathbf{x}=\mathbf{u} \mathbf{T}^{\mathrm{T}}+k \mathbf{X} \mathbf{R}^{\mathrm{T}}
$$

The data are nor error free and this fact is taken into account by adding the error matrix $\mathbf{E}$. Thus, the Procrustes final equation is:

$$
\mathbf{x}=\mathbf{u} \mathbf{T}^{\mathrm{T}}+k \mathbf{X} \mathbf{R}^{\mathrm{T}}+\mathbf{E}
$$

The conditions imposed to determine the parameters of the transformation are:

- Norm of $\mathbf{E}$ must be a minimum.

- $\quad \mathbf{R}$ is orthogonal

Since we need to solve a conditioned minimum, among all the possible solutions, the Lagrange method is considered. The norm used will be the Frobenius Norm:

Thus, the equation to be solved is (3) with the conditions:

$$
\|\mathbf{E}\|_{P}=\min . \quad \mathbf{R}^{\mathrm{T}} \mathbf{R}=1 \text { and } \quad \operatorname{det}(\mathbf{R})=+1
$$

The Frobenius norm (seminorm) is defined by means of the trace of matrix as follows

$$
\|\mathbf{E}\|_{P}^{2}=\operatorname{tr}\left(\mathbf{E}^{\mathrm{T}} \mathbf{P E}\right)
$$

The weight matrix $\mathbf{P}$ of the observations is semidefinite positive because it might occur that not all the elements are known. In this cases, the solution is obtained by using null values for such element. $\mathbf{P}$ is computed by taking into account the dispersion matrixes of both sets of coordinates (see sec. 3.3).

To solve (3), subject to conditions (11) the first step is to compute the translation vector $\mathbf{T}$. The norm to be minimized is

$$
\begin{aligned}
\mathrm{N}(k, \mathbf{T}, \mathbf{R})= & \operatorname{tr}\left(\mathbf{x}-\mathbf{u} \mathbf{T}^{\mathrm{t}}-k \mathbf{X} \mathbf{R}^{\mathrm{T}}\right)^{\mathrm{T}} \mathbf{P}\left(\mathbf{x}-\mathbf{u} \mathbf{T}^{\mathrm{T}}-k \mathbf{X} \mathbf{R}^{\mathrm{T}}\right)= \\
= & \operatorname{tr}\left(\mathbf{x}^{\mathrm{T}} \mathbf{P} \mathbf{x}-\mathbf{x}^{\mathrm{T}} \mathbf{P} \mathbf{u} \mathbf{T}^{\mathrm{T}}-k \mathbf{x}^{\mathrm{T}} \mathbf{P} \mathbf{X} \mathbf{R}^{\mathrm{T}}-\right. \\
& -\mathbf{T} \mathbf{u}^{\mathrm{T}} \mathbf{P} \mathbf{x}+\mathbf{T} \mathbf{u}^{\mathrm{T}} \mathbf{P} \mathbf{u} \mathbf{T}^{\mathrm{T}}+k \mathbf{T} \mathbf{u}^{\mathrm{T}} \mathbf{P} \mathbf{X} \mathbf{R}^{\mathrm{T}}- \\
& \left.-k \mathbf{R} \mathbf{X}^{\mathrm{T}} \mathbf{P} \mathbf{x}+k \mathbf{R} \mathbf{X}^{\mathrm{T}} \mathbf{P} \mathbf{u} \mathbf{T}^{\mathrm{T}}+k^{2} \mathbf{R} \mathbf{X}^{\mathrm{T}} \mathbf{P} \mathbf{X} \mathbf{R}^{\mathrm{T}}\right)
\end{aligned}
$$

For (13) to be a minimum, its partial derivative with respect to the unknowns must be null:

$$
\begin{aligned}
\frac{\partial \mathrm{N}}{\partial \mathbf{T}^{\mathrm{T}}} & =-\mathbf{x}^{\mathrm{T}} \mathbf{P} \mathbf{u}-\mathbf{x}^{\mathrm{T}} \mathbf{P} \mathbf{u}+2 \mathbf{T} \mathbf{u}^{\mathrm{T}} \mathbf{P} \mathbf{u}+k \mathbf{R} \mathbf{X}^{\mathrm{T}} \mathbf{P} \mathbf{u}+k \mathbf{R} \mathbf{X}^{\mathrm{T}} \mathbf{P u}= \\
& =-2 \mathbf{x}^{\mathrm{T}} \mathbf{P} \mathbf{u}+2 \mathbf{T} \mathbf{u}^{\mathrm{T}} \mathbf{P} \mathbf{u}+2 k \mathbf{R} \mathbf{X}^{\mathrm{T}} \mathbf{P} \mathbf{u}=0
\end{aligned}
$$

Finally, the translation vector can be written as:

$$
\mathbf{T}=\left(\mathbf{u}^{\mathrm{T}} \mathbf{P} \mathbf{u}\right)^{-1}\left(\mathbf{x}^{\mathrm{T}}-k \mathbf{R} \mathbf{X}^{\mathrm{T}}\right) \mathbf{P} \mathbf{u}
$$

In the special case that $\mathbf{P}=\mathbf{I}$, the solution is :

$$
\mathbf{T}=\frac{1}{n}\left(\mathbf{x}^{\mathrm{T}}-k \mathbf{R} \mathbf{X}^{\mathrm{T}}\right) \mathbf{u}=\frac{1}{n}\left(\mathbf{x}-k \mathbf{X} \mathbf{R}^{\mathrm{T}}\right)^{\mathrm{T}} \mathbf{u}
$$

Once the translation vector has been computed, we do now proceed to obtain the scale factor.

Taking into account (15), the equation to be solved is now:

$$
\begin{gathered}
\mathrm{N}(k, \mathbf{R})=\operatorname{tr}\left[\mathbf{x}-\mathbf{u u}^{\mathrm{T}} \mathbf{P}\left(\mathbf{x}^{\mathrm{T}}-k \mathbf{R} \mathbf{X}^{\mathrm{T}}\right)^{\mathrm{T}}\left(\mathbf{u}^{\mathrm{T}} \mathbf{P} \mathbf{u}\right)^{-1}-k \mathbf{X} \mathbf{R}^{\mathrm{T}}\right]^{\mathrm{T}} \mathbf{P} \\
{\left[\mathbf{x}-\mathbf{u} \mathbf{u}^{\mathrm{T}} \mathbf{P}\left(\mathbf{x}^{\mathrm{T}}-k \mathbf{R} \mathbf{X}^{\mathrm{T}}\right)^{\mathrm{T}}\left(\mathbf{u}^{\mathrm{T}} \mathbf{P} \mathbf{u}\right)^{-1}-k \mathbf{X} \mathbf{R}^{\mathrm{T}}\right]}
\end{gathered}
$$

(17) is centred because the translation parameters have been included in the formula.

$$
\begin{aligned}
\mathrm{N}(k, \mathbf{R})= & \operatorname{tr}\left[\left(\mathbf{x}-k \mathbf{X} \mathbf{R}^{\mathrm{T}}\right)-\left(\mathbf{u}^{\mathrm{T}} \mathbf{P} \mathbf{u}\right)^{-1} \mathbf{u} \mathbf{u}^{\mathrm{T}} \mathbf{P}\left(\mathbf{x}-k \mathbf{X} \mathbf{R}^{\mathrm{T}}\right)\right]^{\mathrm{T}} \mathbf{P} \\
& {\left[\left(\mathbf{x}-k \mathbf{X} \mathbf{R}^{\mathrm{T}}\right)-\left(\mathbf{u}^{\mathrm{T}} \mathbf{P} \mathbf{u}\right)^{-1} \mathbf{u} \mathbf{u}^{\mathrm{T}} \mathbf{P}\left(\mathbf{x}-k \mathbf{X} \mathbf{R}^{\mathrm{T}}\right)\right] } \\
= & \operatorname{tr}\left\{\left[\mathbf{I}-\left(\mathbf{u}^{\mathrm{T}} \mathbf{P} \mathbf{u}\right)^{-1} \mathbf{u} \mathbf{u}^{\mathrm{T}} \mathbf{P}\right]\left(\mathbf{x}-k \mathbf{X} \mathbf{R}^{\mathrm{T}}\right)\right\}^{\mathrm{T}} \mathbf{P} . \\
& \cdot\left\{\left[\mathbf{I}-\left(\mathbf{u}^{\mathrm{T}} \mathbf{P} \mathbf{u}\right)^{-1} \mathbf{u} \mathbf{u}^{\mathrm{T}} \mathbf{P}\right]\left(\mathbf{x}-k \mathbf{X} \mathbf{R}^{\mathrm{T}}\right)\right\}
\end{aligned}
$$

A new matrix shall be defined:

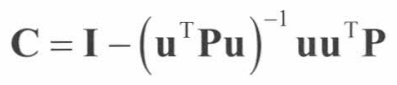

With (19), (18) can now be written as:

$$
\begin{aligned}
\mathrm{N}(k, \mathbf{R}) & =\operatorname{tr}\left\{\left[\mathbf{C}\left(\mathbf{x}-k \mathbf{X} \mathbf{R}^{\mathrm{T}}\right)\right]^{\mathrm{T}} \mathbf{P}\left[\mathbf{C}\left(\mathbf{x}-k \mathbf{X} \mathbf{R}^{\mathrm{T}}\right)\right]\right\} \\
& =\operatorname{tr}\left\{\left(\mathbf{x}^{\mathrm{T}}-k \mathbf{R} \mathbf{X}^{\mathrm{T}}\right) \mathbf{C}^{\mathrm{T}} \mathbf{P} \mathbf{C}\left(\mathbf{x}-k \mathbf{X} \mathbf{R}^{\mathrm{T}}\right)\right\}
\end{aligned}
$$

or

$$
\mathrm{N}(k, \mathbf{R})=\operatorname{tr}\left(\begin{array}{l}
\mathbf{x}^{\mathrm{T}} \mathbf{C}^{\mathrm{T}} \mathbf{P C} \mathbf{C}-k \mathbf{x}^{\mathrm{T}} \mathbf{C}^{\mathrm{T}} \mathbf{P} \mathbf{C X} \mathbf{R}^{\mathrm{T}}- \\
-k \mathbf{R} \mathbf{X}^{\mathrm{T}} \mathbf{C}^{\mathrm{T}} \mathbf{P} \mathbf{C} \mathbf{x}+k^{2} \mathbf{R} \mathbf{X}^{\mathrm{T}} \mathbf{C}^{\mathrm{T}} \mathbf{P} \mathbf{C} \mathbf{X} \mathbf{R}^{\mathrm{T}}
\end{array}\right)
$$


Eq. (21) must be a minimum, so its derivative with respect the unknown must be null:

$$
\begin{array}{r}
\frac{\partial \mathrm{N}(k, \mathbf{R})}{\partial k}=-2 \operatorname{tr}\left(\mathbf{x}^{\mathrm{T}} \mathbf{C}^{\mathrm{T}} \mathbf{P} \mathbf{C X \mathbf { R } ^ { \mathrm { T } } ) +}\right. \\
+2 k \operatorname{tr}\left(\mathbf{R} \mathbf{X}^{\mathrm{T}} \mathbf{C}^{\mathrm{T}} \mathbf{P} \mathbf{C} \mathbf{X} \mathbf{R}^{\mathrm{T}}\right)=0
\end{array}
$$

Finally, we get the scale factor:

$$
k=\frac{\operatorname{tr}\left(\mathbf{x}^{\mathrm{T}} \mathbf{C}^{\mathrm{T}} \mathbf{P} \mathbf{C X \mathbf { R } ^ { \mathrm { T } } )}\right.}{\operatorname{tr}\left(\mathbf{X}^{\mathrm{T}} \mathbf{C}^{\mathrm{T}} \mathbf{P C X}\right)}
$$

In the particular case that the weight matrix is diagonal unitary, the scale factor obtained is:

$$
\begin{aligned}
\mathbf{C} & =\mathbf{I}-\frac{1}{n} \mathbf{u} \mathbf{u}^{\mathrm{T}} \\
k & =\frac{\operatorname{tr}\left(\mathbf{x}^{\mathrm{T}} \mathbf{C} \mathbf{X} \mathbf{R}^{\mathrm{T}}\right)}{\operatorname{tr}\left(\mathbf{X}^{\mathrm{T}} \mathbf{C X}\right)}
\end{aligned}
$$

Once the translation vector and the scale factor have been computes, the rotation matrix will be computed. To compute the rotations, (17) and (23) are taken into account. Thus, the new equation to be minimized is:

$$
\begin{aligned}
& \mathrm{N}(\mathbf{R})=\operatorname{tr}\left(\mathbf{x}^{\mathrm{T}}-\frac{\operatorname{tr}\left(\mathbf{x}^{\mathrm{T}} \mathbf{C}^{\mathrm{T}} \mathbf{P C} \mathbf{X} \mathbf{R}^{\mathrm{T}}\right)}{\operatorname{tr}\left(\mathbf{X}^{\mathrm{T}} \mathbf{C}^{\mathrm{T}} \mathbf{P C} \mathbf{X}\right)} \mathbf{R X}^{\mathrm{T}}\right) \mathbf{C}^{\mathrm{T}} \mathbf{P C}\left(\mathbf{x}-\frac{\operatorname{tr}\left(\mathbf{x}^{\mathrm{T}} \mathbf{C}^{\mathrm{T}} \mathbf{P} \mathbf{C} \mathbf{X} \mathbf{R}^{\mathrm{T}}\right)}{\operatorname{tr}\left(\mathbf{X}^{\mathrm{T}} \mathbf{C}^{\mathrm{T}} \mathbf{P C} \mathbf{X}\right)} \mathbf{X R}^{\mathrm{T}}\right) \\
& =\operatorname{tr}\left(\mathbf{x}^{\mathrm{T}} \mathbf{C}^{\mathrm{T}} \mathbf{P C} \mathbf{x}\right)-\frac{\operatorname{tr}\left(\mathbf{x}^{\mathrm{T}} \mathbf{C}^{\mathrm{T}} \mathbf{P C} \mathbf{C} \mathbf{R}^{\mathrm{T}}\right)}{\operatorname{tr}\left(\mathbf{X}^{\mathrm{T}} \mathbf{C}^{\mathrm{T}} \mathbf{P C X}\right)} \operatorname{tr}\left(\mathbf{x}^{\mathrm{T}} \mathbf{C}^{\mathrm{T}} \mathbf{P C} \mathbf{C} \mathbf{R}^{\mathrm{T}}\right)- \\
& -\frac{\operatorname{tr}\left(\mathbf{x}^{\mathrm{T}} \mathbf{C}^{\mathrm{T}} \mathbf{P C X} \mathbf{R}^{\mathrm{T}}\right)}{\operatorname{tr}\left(\mathbf{X}^{\mathrm{T}} \mathbf{C}^{\mathrm{T}} \mathbf{P C X}\right)} \operatorname{tr}\left(\mathbf{R} \mathbf{X}^{\mathrm{T}} \mathbf{C}^{\mathrm{T}} \mathbf{P C} \mathbf{x}\right)+ \\
& +\frac{\operatorname{tr}\left(\mathbf{x}^{\mathrm{T}} \mathbf{C}^{\mathrm{T}} \mathbf{P} \mathbf{C} \mathbf{X} \mathbf{R}^{\mathrm{T}}\right)}{\operatorname{tr}\left(\mathbf{X}^{\mathrm{T}} \mathbf{C}^{\mathrm{T}} \mathbf{P C X}\right)} \frac{\operatorname{tr}\left(\mathbf{x}^{\mathrm{T}} \mathbf{C}^{\mathrm{T}} \mathbf{P} \mathbf{C} \mathbf{X} \mathbf{R}^{\mathrm{T}}\right)}{\operatorname{tr}\left(\mathbf{X}^{\mathrm{T}} \mathbf{C}^{\mathrm{T}} \mathbf{P C X}\right)} \operatorname{tr}\left(\mathbf{R X}^{\mathrm{T}} \mathbf{C}^{\mathrm{T}} \mathbf{P} \mathbf{C} \mathbf{X} \mathbf{R}^{\mathrm{T}}\right) \\
& =\operatorname{tr}\left(\mathbf{x}^{\mathrm{T}} \mathbf{C}^{\mathrm{T}} \mathbf{P C}\right)-2 \frac{\operatorname{tr}\left(\mathbf{x}^{\mathrm{T}} \mathbf{C}^{\mathrm{T}} \mathbf{P} \mathbf{C} \mathbf{X} \mathbf{R}^{\mathrm{T}}\right)^{2}}{\operatorname{tr}\left(\mathbf{X}^{\mathrm{T}} \mathbf{C}^{\mathrm{T}} \mathbf{P C}\right)}+\frac{\operatorname{tr}\left(\mathbf{x}^{\mathrm{T}} \mathbf{C}^{\mathrm{T}} \mathbf{P} \mathbf{C} \mathbf{X} \mathbf{R}^{\mathrm{T}}\right)^{2}}{\operatorname{tr}\left(\mathbf{X}^{\mathrm{T}} \mathbf{C}^{\mathrm{T}} \mathbf{P C} \mathbf{X}\right)} \\
& =\operatorname{tr}\left(\mathbf{x}^{\mathrm{T}} \mathbf{C}^{\mathrm{T}} \mathbf{P C} \mathbf{x}\right)-\frac{\operatorname{tr}\left(\mathbf{x}^{\mathrm{T}} \mathbf{C}^{\mathrm{T}} \mathbf{P C} \mathbf{C} \mathbf{R}^{\mathrm{T}}\right)^{2}}{\operatorname{tr}\left(\mathbf{X}^{\mathrm{T}} \mathbf{C}^{\mathrm{T}} \mathbf{P C X}\right)}
\end{aligned}
$$

The condition for (25) to be a minimum is:

$$
\operatorname{tr}\left(\mathbf{x}^{\mathrm{T}} \mathbf{C}^{\mathrm{T}} \mathbf{P} \mathbf{C X} \mathbf{R}^{\mathrm{T}}\right)=\max
$$

Let $\mathbf{D}=\mathbf{x}^{\mathrm{T}} \mathbf{C}^{\mathrm{T}} \mathbf{P C X}$ and let $\mathbf{U} \boldsymbol{\Sigma} \mathbf{V}^{\mathrm{T}}$ be its Singular Value Decomposition. Then,

$$
\begin{aligned}
& \mathbf{U} \mathbf{U}^{\mathrm{T}}=\mathbf{U}^{\mathrm{T}} \mathbf{U}=\mathbf{I} \\
& \mathbf{V} \mathbf{V}^{\mathrm{T}}=\mathbf{V}^{\mathrm{T}} \mathbf{V}=\mathbf{I} \\
& \boldsymbol{\Sigma}=\operatorname{diagonal}\left(\sigma_{1}, \sigma_{2}, \ldots, \sigma_{n}\right)
\end{aligned}
$$

We can now write:

$$
\mathbf{x}^{\mathrm{T}} \mathbf{C}^{\mathrm{T}} \mathbf{P C X} \mathbf{R}^{\mathrm{T}}=\mathbf{D} \mathbf{R}^{\mathrm{T}}=\mathbf{U} \boldsymbol{\Sigma} \mathbf{V}^{\mathrm{T}} \mathbf{R}^{\mathrm{T}}
$$

Now:

$$
\begin{aligned}
\operatorname{tr}\left(\mathbf{x}^{\mathrm{T}} \mathbf{C}^{\mathrm{T}} \mathbf{P} \mathbf{C} \mathbf{X} \mathbf{R}^{\mathrm{T}}\right) & =\operatorname{tr}\left(\mathbf{D} \mathbf{R}^{\mathrm{T}}\right) \\
\operatorname{tr}\left(\mathbf{U} \boldsymbol{\Sigma} \mathbf{V}^{\mathrm{T}} \mathbf{R}^{\mathrm{T}}\right) & =\operatorname{tr}\left(\boldsymbol{\Sigma} \mathbf{V}^{\mathrm{T}} \mathbf{R}^{\mathrm{T}} \mathbf{U}\right)
\end{aligned}
$$

let $\mathbf{K}=\mathbf{V}^{\mathrm{T}} \mathbf{R}^{\mathrm{T}} \mathbf{U}$, which is an orthogonal matrix with diagonal entries such that $\left|k_{i i}\right| \leq 1$, then

$$
\operatorname{tr}\left(\boldsymbol{\Sigma} \mathbf{V}^{\mathrm{T}} \mathbf{R}^{\mathrm{T}} \mathbf{U}\right)=\operatorname{tr}(\boldsymbol{\Sigma} \mathbf{K})=\sum_{i=1}^{n} \sigma_{i} k_{i i} \leq \sum_{i=1}^{n} \sigma_{i}
$$

The maximum value of (30) is given by:

$$
\begin{aligned}
& \max \left(\operatorname{trx}^{\mathrm{T}} \mathbf{C}^{\mathrm{T}} \mathbf{P} \mathbf{C X \mathbf { R } ^ { \mathrm { T } }}\right)=\max (\operatorname{tr} \mathbf{\Sigma} \mathbf{K}) \\
& =\sum_{i=1}^{n} \sigma_{i}=\operatorname{tr} \Sigma
\end{aligned}
$$

But the maximum is:

$$
\operatorname{tr}\left(\boldsymbol{\Sigma} \mathbf{V}^{\mathrm{T}} \mathbf{R}^{\mathrm{T}} \mathbf{U}\right)=\operatorname{tr} \boldsymbol{\Sigma},
$$

so that

$$
\mathbf{V}^{\mathrm{T}} \mathbf{R}^{\mathrm{T}} \mathbf{U}=\mathbf{I}
$$

Multiplying by $\mathbf{V}$ and $\mathbf{U}^{\mathrm{T}}$, we get:

$$
\begin{aligned}
& \mathbf{V} \mathbf{V}^{\mathrm{T}} \mathbf{R}^{\mathrm{T}} \mathbf{U}=\mathbf{V} \\
& \mathbf{R}^{\mathrm{T}} \mathbf{U}=\mathbf{V} \\
& \mathbf{R}^{\mathrm{T}} \mathbf{U} \mathbf{U}^{\mathrm{T}}=\mathbf{V} \mathbf{U}^{\mathrm{T}}
\end{aligned}
$$

From (34) we can write:

$$
\mathbf{R}=\mathbf{U} \mathbf{V}^{\mathbf{T}}
$$

For the special case that $\mathbf{P}=\mathbf{I}$, we get:

$$
\begin{aligned}
& \mathbf{D}=\mathbf{x}^{\mathrm{T}} \mathbf{C} \mathbf{X}=\mathbf{U} \boldsymbol{\Sigma} \mathbf{V}^{\mathrm{T}} \\
& \mathbf{R}=\mathbf{U} \mathbf{V}^{\mathbf{T}}
\end{aligned}
$$

We can now compute the residuals by taking into account all the parameters computed:

$$
\mathbf{E}=\left(\mathbf{I}-\left(\mathbf{u}^{\mathrm{T}} \mathbf{P u}\right)^{-1} \mathbf{u} \mathbf{u}^{\mathrm{T}} \mathbf{P}\right)\left(\mathbf{x}-\frac{\operatorname{tr}\left(\mathbf{x}^{\mathrm{T}} \mathbf{C}^{\mathrm{T}} \mathbf{P} \mathbf{C} \mathbf{X V} \mathbf{U}^{\mathrm{T}}\right)}{\operatorname{tr}\left(\mathbf{X}^{\mathrm{T}} \mathbf{C}^{\mathrm{T}} \mathbf{P C X}\right)} \mathbf{X V} \mathbf{U}^{\mathrm{T}}\right)
$$


The Frobenius norm, which can be considered as a measure of the quality of the transformation equals:

$\|E\|_{\mathbf{P}}^{2}=\operatorname{tr}\left[\left(\mathbf{I}-\left(\mathbf{u}^{\top} \mathbf{P u}\right)^{-1} \mathbf{u} \mathbf{u}^{\mathrm{T}} \mathbf{P}\right)\left(\mathbf{x}-\frac{\operatorname{tr}\left(\mathbf{x}^{\mathrm{T}} \mathbf{C}^{\mathrm{T}} \mathbf{P} \mathbf{C} \mathbf{X V} \mathbf{U}^{\mathrm{T}}\right)}{\operatorname{tr}\left(\mathbf{X}^{\mathrm{T}} \mathbf{C}^{\mathrm{T}} \mathbf{P C X}\right)} \mathbf{X V} \mathbf{U}^{\mathrm{T}}\right)\right]^{\mathrm{T}} \mathbf{P}$
$\left[\left(\mathbf{I}-\left(\mathbf{u}^{\mathrm{T}} \mathbf{P u}\right)^{-1} \mathbf{u} \mathbf{u}^{\mathrm{T}} \mathbf{P}\right)\left(\mathbf{x}-\frac{\operatorname{tr}\left(\mathbf{x}^{\mathrm{T}} \mathbf{C}^{\mathrm{T}} \mathbf{P} \mathbf{C} \mathbf{X V} \mathbf{U}^{\mathrm{T}}\right)}{\operatorname{tr}\left(\mathbf{X}^{\top} \mathbf{C}^{\mathrm{T}} \mathbf{P C X}\right)} \mathbf{X V} \mathbf{U}^{\mathrm{T}}\right)\right]$

The values for the residuals and the Frobenius Norm, in the special case $\mathbf{P}=\mathbf{I}$ are respectively:

$$
\begin{gathered}
\mathbf{E}=\left(\mathbf{I}-\frac{1}{n} \mathbf{u u}^{\mathrm{T}}\right)\left(\mathbf{x}-\frac{\operatorname{tr}\left(\mathbf{x}^{\mathrm{T}} \mathbf{C} \mathbf{X} \mathbf{V} \mathbf{U}^{\mathrm{T}}\right)}{\operatorname{tr}\left(\mathbf{X}^{\mathrm{T}} \mathbf{C} \mathbf{X}\right)} \mathbf{X} \mathbf{V} \mathbf{U}^{\mathrm{T}}\right) \\
\|\mathrm{E}\|_{\mathrm{P}}^{2}=\operatorname{tr}\left(\mathbf{x}-\frac{\operatorname{tr}\left(\mathbf{x}^{\mathrm{T}} \mathbf{C} \mathbf{X V} \mathbf{U}^{\mathrm{T}}\right)}{\operatorname{tr}\left(\mathbf{X}^{\mathrm{T}} \mathbf{C X}\right)} \mathbf{X V \mathbf { U } ^ { \mathrm { T } }}\right)^{\mathrm{T}}\left(\mathbf{I}-\frac{1}{n} \mathbf{u} \mathbf{u}^{\mathrm{T}}\right) \\
\left(\mathbf{x}-\frac{\operatorname{tr}\left(\mathbf{x}^{\mathrm{T}} \mathbf{C} \mathbf{X V} \mathbf{U}^{\mathrm{T}}\right)}{\operatorname{tr}\left(\mathbf{X}^{\mathrm{T}} \mathbf{C} \mathbf{X}\right)} \mathbf{X V U}^{\mathrm{T}}\right)
\end{gathered}
$$

\subsection{Computation of the Weight Matrix}

The Weighted Procrustes solution takes into account the dispersions of both coordinate sets. Nevertheless, the weight matrix $P$, has not been defined yet. This section deals with the computation of the weight matrix.

The weight matrix must take into account the dispersion matrices of both sets of coordinates and, of course, the local dispersion matrix when it is transformed to the global frame. It has been proved (Awange and Grafarend., 2005) that the weight matrix equals to:

$$
\begin{aligned}
\Sigma_{v e c \mathbf{E}^{\mathrm{T}}} & =\Sigma_{v e c \mathbf{x}^{\mathrm{T}}}+\left(\mathbf{I}_{n} \otimes k \mathbf{R}\right) \Sigma_{v e c \mathbf{X}^{\mathrm{T}}}\left(\mathbf{I}_{n} \otimes k \mathbf{R}\right)^{\mathrm{T}}- \\
& -2 \Sigma_{v e c \mathbf{X}^{\mathrm{T}},\left(\mathbf{I}_{n} \otimes k \mathbf{R}\right) \operatorname{vec} \mathbf{X}^{\mathrm{T}}}
\end{aligned}
$$

In (41), $\Sigma$ makes reference to the variance covariance matrix, vec is the vec operator and $\otimes$ is the Kronecker product.

\subsection{Partial Procrustes Analysis}

The partial Procrustes Analysis deals with the computation only of the rotation elements. No translation vector nor scale factor are computed (Awange and Grafarend, 2005). Thus, the solution is given by (35) and (36).
Once the rotations matrix is computed, the next step is to transform the matrix elements into the orientation elements: astronomic latitude $(\Phi)$ astronomic longitude $(\Lambda)$ and the orientation unknown $(\Sigma)$, as has been stated in section 3, Euler angles are obtained by the parameterization of the rotation matrix as follows:

$$
\mathbf{R}(\Lambda, \Phi, \Sigma)=\mathbf{R}(\Sigma) \cdot \mathbf{R}\left(\frac{\pi}{2}-\Phi\right) \cdot \mathbf{R}(\Lambda)
$$

Taking into account the general rotations matrix for each angle, substituting the orientation angles and multiplying in the order (42), we get:

$$
\begin{aligned}
& \mathbf{R}=\mathbf{R}(\Sigma) \mathbf{R}\left(\frac{\pi}{2}-\Phi\right) \mathbf{R}(\Lambda) \\
& =\left(\begin{array}{ccc}
\cos (\Sigma) \sin (\Phi) \cos (\Lambda)- & \cos (\Sigma) \sin (\Phi) \sin (\Lambda)+ & -\cos (\Sigma) \cos (\Phi) \\
-\sin (\Sigma) \sin (\Lambda) & +\sin (\Sigma) \cos (\Lambda) & \\
-\sin (\Sigma) \sin (\Phi) \cos (\Lambda)- & -\sin (\Sigma) \sin (\Phi) \sin (\Lambda)+ & \sin (\Sigma) \cos (\Phi) \\
-\cos (\Sigma) \sin (\Lambda) & +\cos (\Sigma) \cos (\Lambda) & \sin (\Phi) \\
\cos (\Phi) \cos (\Lambda) & \cos (\Phi) \sin (\Lambda) &
\end{array}\right.
\end{aligned}
$$

\section{Case Study}

The data sets (courtesy of the Gipuzkoako Foru Aldundia: b5m.gipuzkoa.net) correspond to the GPS network of Gipuzkoa, which is formed by a total of 21 stations which coordinates are known in the ITRF00 frame and, thus, in the ETRS89 Reference System by applying the Altamimi transformation parameters (Altamimi et al. 2002).

To verify the capability of the WEOP to detect deformations different movements have been simulated in 6 stations. The displacements vary from 1 to $6 \mathrm{~cm}$ in the $\mathrm{X}$ and in the $\mathrm{Z}$ components. The movements simulated have been: ITURREGI $(1 \mathrm{~cm})$, MENDIZORROTZ $(2 \mathrm{~cm})$, ONDDY (3 $\mathrm{cm})$, BIANDITZ $(4 \mathrm{~cm})$, ZUBELTZU $(5 \mathrm{~cm})$ and SAN TELMO $(6 \mathrm{~cm})$. The analysis of the residuals should tell us if the WEOP is or not a valid model to detect deformations. Real data has been used.

The residuals will be compared with those obtained from the classical Helmert datum transformation.

Both systems are global. The network (Figure 3) was stabilised in 2002 and it covers completely the Territory of Gipuzkoa, which is located in the Basque Country (Northern Spain) and its surface is about $2000 \mathrm{~km}^{2}$. The network consists in 21 station which belong to the "Active and Passive GPS Network of Gipuzkoa" and it was presented in the 
"4 $4^{\mathrm{a}}$ Asamblea Hispanolusa de Geodesia y Geofísica", February 2004, Figueira da Foz (Portugal) (Zurutuza et al., 2004). Each station has been occupied at least twice in separate sessions of not less than 8 hours of time span. A total of 7 dual frequency GPS receptors were used in each session. The 15 sessions were computed taking into account only the non trivial vectors. After the adjustment of the whole campaign, the global accuracy was estimated to be better than $2 \mathrm{~cm}$ with respect the active station. The coordinates of the Active Station were also computed and the accuracy estimated was better than $1,5 \mathrm{~cm}$ in the ITRF00 frame. Once the Reference Station was computed, the rest of the stations were adjusted constraining strongly the reference one.

The main processing parameters were: a. Elevation Mask: $10^{\circ}$.

b. Precise IGS ephemeris.

c. NO ocean loading model..

d. Tropospheric model: Niell model (Niell, 1996)

e. Ambiguities: QIF strategy (Hugentobler et al., 2001).

f. Processing frequency: $\mathrm{L}_{3}$.

g. Software package: Bernese V 4.2.

The first part of the problem shows the differences between the classical and the WEOP transformations, while the second part deals with the deformation detection.

In table 1 the Cartesian coordinates of both sets are presented. Table 2 shows the computed coordinates after the transformation

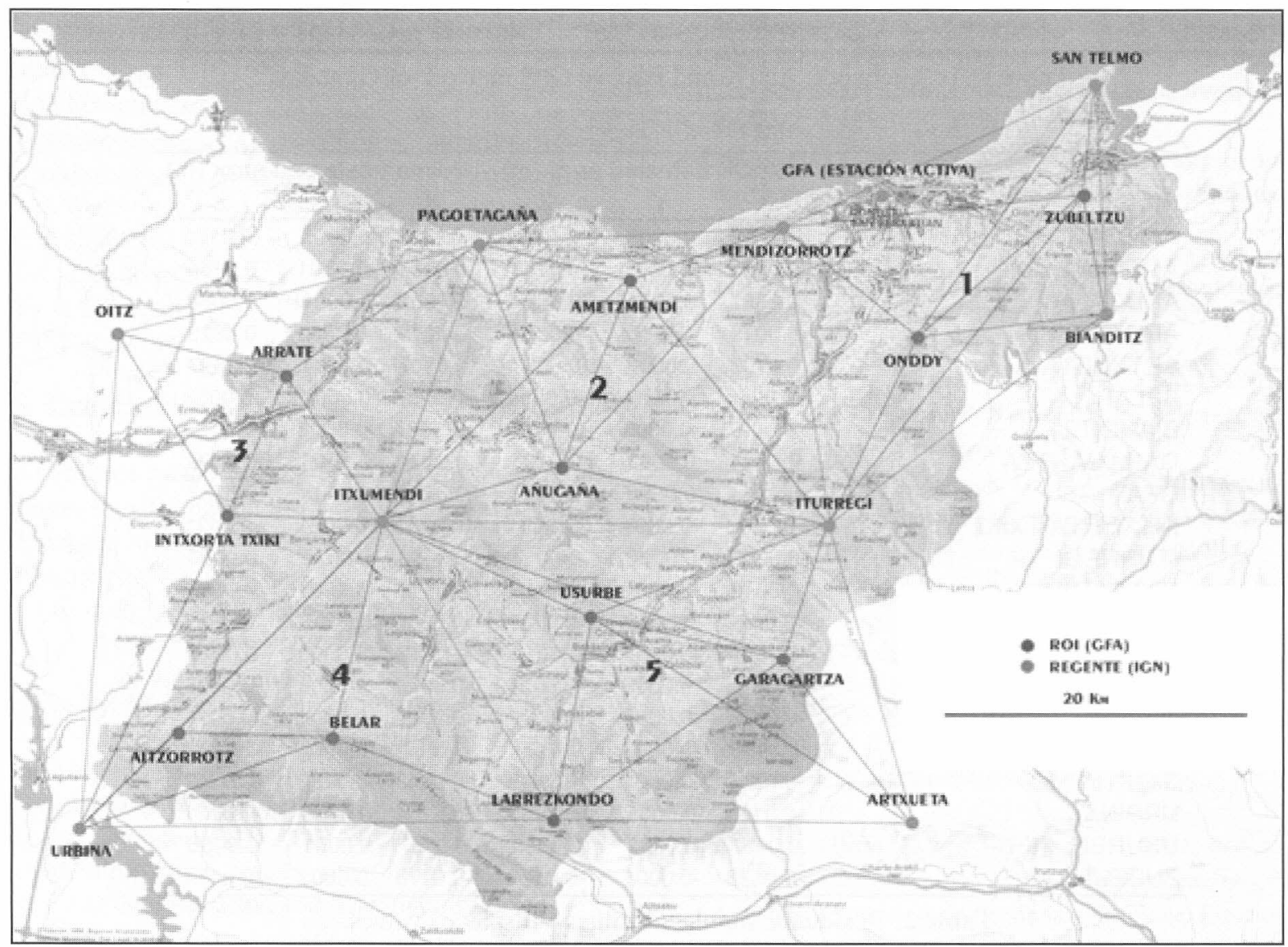

Fig 3: Test network: Active and Passive GPS network of Gipuzkoa. 


\begin{tabular}{lcccccc}
\hline & \multicolumn{3}{c}{ ITRF00 Coordinates $(\mathrm{m})$} & & \multicolumn{3}{c}{ ETRS89 Coordinates (m) } \\
Station. & $X$ & $Y$ & $Z$ & X & Y & Z \\
\hline AITZORROTZ & 4667813.0044 & -207091.2377 & 4328176.0110 & 4667813.1903 & -207091.4595 & 4328175.8077 \\
AMETZMENDI & 4648581.5707 & -176745.6283 & 4349215.1022 & 4648581.7589 & -176745.8491 & 4349214.8997 \\
ANUGANA & 4656972.4871 & -181645.8861 & 4340292.5540 & 4656972.6747 & -181646.1073 & 4340292.3512 \\
ARRATE & 4652438.4412 & -199058.1997 & 4344726.7660 & 4652438.6280 & -199058.4207 & 4344726.5633 \\
ARTXUETA & 4673825.5758 & -160365.1493 & 4324588.3343 & 4673825.7640 & -160365.3714 & 4324588.1311 \\
BELAR & 4668121.4190 & -197465.2942 & 4328524.5982 & 4668121.6054 & -197465.5160 & 4328524.3950 \\
BIANDITZ & 4651583.8997 & -145430.0779 & 4348133.1014 & 4651584.0495 & -145430.2989 & 4348132.8590 \\
GARAGARTZA & 4664840.1331 & -169153.4995 & 4332432.9138 & 4664840.3211 & -169153.7211 & 4332432.7108 \\
GFA & 4644833.0652 & -160817.6784 & 4353575.0764 & 4644833.2544 & -160817.8991 & 4353574.8741 \\
INTXORTA TXIKI & 4658356.6606 & -202888.2927 & 4338445.1462 & 4658356.8470 & -202888.5140 & 4338444.9433 \\
ITURREGI & 4660182.6604 & -164249.4372 & 4338251.9550 & 4660182.8389 & -164249.6586 & 4338251.7422 \\
ITXUMENDI & 4658830.9201 & -193130.1434 & 4338396.6208 & 4658831.1070 & -193130.3647 & 4338396.4179 \\
LARREZKONDO & 4672777.6644 & -182116.7758 & 4323931.8770 & 4672777.8515 & -182116.9978 & 4323931.6737 \\
MENDIZORROTZ & 4646636.3737 & -167697.9967 & 4351962.2560 & 4646636.5425 & -167698.2175 & 4351962.0336 \\
OITZ & 4650697.7378 & -210481.1412 & 4346696.1158 & 4650697.9241 & -210481.3621 & 4346695.9131 \\
ONDDY & 4652015.6655 & -158468.8661 & 4346786.2290 & 4652015.8246 & -158469.0871 & 4346785.9965 \\
PAGOETAGANA & 4646548.0436 & -185892.9832 & 4351053.8710 & 4646548.2314 & -185893.2039 & 4351053.6686 \\
SAN TELMO & 4640529.6294 & -145676.2974 & 4358781.6667 & 4640529.7596 & -145676.5179 & 4358781.4047 \\
URBINA & 4671792.8232 & -213717.2423 & 4323528.7964 & 4671793.0086 & -213717.4642 & 4323528.5930 \\
USURBE & 4664072.7258 & -179486.9185 & 4333355.0737 & 4664072.9133 & -179487.1401 & 4333354.8707 \\
ZUBELTZU & 4645449.6443 & -146437.3727 & 4353693.2581 & 4645449.7842 & -146437.5934 & 4353693.0059 \\
\hline
\end{tabular}

Table 1: Test network: Active and Passive GPS network of Gipuzkoa.

\begin{tabular}{|c|c|c|c|c|c|c|c|}
\hline \multirow[b]{2}{*}{ Station } & \multirow[b]{2}{*}{ Error $(\mathrm{cm})$ : } & \multicolumn{3}{|c|}{ WEOP detected error (m) } & \multicolumn{3}{|c|}{ Helmert detected error (m) } \\
\hline & & $\mathrm{x}$ & $\mathrm{Y}$ & Z & $\mathrm{X}$ & $\mathrm{Y}$ & Z \\
\hline AITZORROTZ & & -0.0108 & 0.0003 & -0.0104 & -0.0107 & 0.0003 & -0.0105 \\
\hline AMETZMENDI & & 0.0153 & -0.0001 & 0.0156 & 0.0153 & -0.0001 & 0.0156 \\
\hline ANUGANA & & 0.0082 & 0.0001 & 0.0084 & 0.0082 & 0.0001 & 0.0084 \\
\hline ARRATE & & 0.0019 & -0.0002 & 0.0022 & 0.0018 & -0.0002 & 0.0022 \\
\hline ARTXUETA & & 0.0107 & 0.0002 & 0.0097 & 0.0107 & 0.0001 & 0.0097 \\
\hline BELAR & & -0.0058 & 0.0002 & -0.0058 & -0.0057 & 0.0002 & -0.0058 \\
\hline BIANDITZ & 4 & -0.0097 & -0.0003 & -0.0103 & -0.0097 & -0.0003 & -0.0103 \\
\hline GARAGARTZA & & 0.0104 & 0.0004 & 0.0103 & 0.0104 & 0.0005 & 0.0103 \\
\hline GFA & & 0.0254 & 0.0000 & 0.0257 & 0.0254 & 0.0000 & 0.0257 \\
\hline INTXORTA TXIKI & & -0.0033 & -0.0001 & -0.0031 & -0.0033 & -0.0001 & -0.0031 \\
\hline ITURREG| & 1 & 0.0058 & 0.0000 & 0.0054 & 0.0058 & 0.0000 & 0.0054 \\
\hline ITXUMENDI & & 0.0016 & -0.0001 & 0.0016 & 0.0015 & -0.0001 & 0.0016 \\
\hline LARREZKONDO & & -0.0005 & 0.0005 & -0.0007 & -0.0005 & 0.0005 & -0.0007 \\
\hline MENDIZORROTZ & 2 & 0.0012 & -0.0002 & 0.0013 & 0.0012 & -0.0003 & 0.0013 \\
\hline OITZ & & -0.0028 & -0.0006 & -0.0026 & -0.0028 & -0.0006 & -0.0026 \\
\hline ONDDY & 3 & -0.0069 & -0.0002 & -0.0071 & -0.0069 & -0.0001 & -0.0071 \\
\hline PAGOETAGANA & & 0.0116 & -0.0002 & 0.0121 & 0.0117 & -0.0002 & 0.0121 \\
\hline SAN TELMO & 6 & -0.0243 & -0.0001 & -0.0243 & -0.0243 & -0.0002 & -0.0242 \\
\hline URBINA & & -0.0165 & 0.0005 & -0.0161 & -0.0164 & 0.0005 & -0.0161 \\
\hline USURBE & & 0.0057 & 0.0002 & 0.0055 & 0.0057 & 0.0001 & 0.0055 \\
\hline ZUBELTZU & 5 & -0.0173 & -0.0001 & -0.0174 & -0.0173 & -0.0001 & -0.0174 \\
\hline
\end{tabular}

Table 2: Residuals: simulated values - displaced values.

As can be seen in tables 1 and 2, the results are the same to the $\mathrm{mm}$ level. This is very important because it means that the WEOP solution is as good as the classical datum transformation. This paper deals with the datum transformation problem though the global to global solution has been tested 
it is also al solution for the local to global or local to local datum transformation.

\subsection{Results}

A very important part of the results has already be shown in table 2. As well as the residuals, it is also interesting to check the parameters obtained:

\section{- Helmert:}

$\begin{array}{lrr}\text { Variance: } & 0.000101895 & \\ \text { Tx } & -2.2890 \mathrm{~m} & 0.8479 \\ \text { Ty } & 4.5539 \mathrm{~m} & 0.7480 \\ \mathrm{Tz} & 2.4625 \mathrm{~m} & 0.8717 \\ \omega x & -5.08316 \mathrm{E}-07 & 1.01058 \mathrm{E}-07 \\ \omega y & -5.7154 \mathrm{E}-07 & 1.7058 \mathrm{E}-07 \\ \omega z & 5.50716 \mathrm{E}-07 & 1.04616 \mathrm{E}-07 \\ \mathrm{k} & 2.23824 \mathrm{E}-08 & 8.57186 \mathrm{E}-08\end{array}$

\section{- WEOP:}

Matrix Norm: 0.075588

Rotation matrix:

$\begin{array}{lll}\left(\begin{array}{lll}0.99999999999969 & -0.00000055053971 & -0.00000057193875 \\ 0.00000055054000 & 0.99999999999972 & 0.00000050783095 \\ 0.00000057193847 & -0.00000050783126 & 0.99999999999971\end{array}\right) \\ \text { k: } 1.00000002191551 & \\ \text { Tx: }-2.2886 \mathrm{~m} & \\ \text { Ty: } 4.55087 \mathrm{~m} & \\ \text { Tz: } 2.46627 \mathrm{~m} & \end{array}$

\section{Conclusions}

A different method, using real data, for the classical datum transformation has been used (Grafarend and Awange, 2003).

The main advantages are:

a. non iterative method, no "a priori" results must be known and it is a straight forward computational method.

b. Method capable of integrating the local and the global reference systems by computing the vertical deflections.

c. No need of linearization and easy to compute.

d. Promising results obtainable in the ITRF determination and densification.

From the authors point of view, the method worth deeper investigation, as well as the inclusion of the stochastic model, which has been already proposed (Awange and Grafarend, 2005)
Helmert vs Procurstes: difficulties comparing rotation results due to the small values obtained in global-to-global transformation.

\section{Acknowledgements}

The authors wish to acknowledge to the people of the Provincial Council of Gipuzkoa the data provided as well as the facilities given to get such data, specially to J. M. Aramburu, M. Elorza, and F. Gainzarain.

\section{References}

Altamimi, Z., P. Sillard and C. Boucher, 2002: ITRF2000: A new release of the International Terrestrial Reference Frame for Earth science applications. J. Geophys. Res. 107, No B10, 2214, 10.1029/2001JB000056.

Awange, J. L., 2003: Partial Procrustes solution of the three dimensional orientation problem from LPS/GPS observations. Geodesy - the Challenge of the 3rd Millenium. Springer, Heidelberg.

Awange, J. L. and E. W Grafarend, 2005. Solving Algebraic Computational Problems in Geodesy and Geoinformatics. Springer.

Crosilla, F (1983): Procrustes transformation as a tool for the construction os a criterion matrix for control networks. Manuscripta Geodaetica 8, pp. 343-370.

Grafarend, E. W. and J. L. Awange (2003): Nonlinear analysis of the three-dimensional datum transformation [conformal group $\boldsymbol{C}_{7}$ (3)]. Journal of Geodesy, 77, pp. 6676

Hugentobler, U., S. Schaer \& P. Fridez (Edts.), 2001: Bernese GPS Software Version 4.2. Astronomical Institute. University of Berne.

Krakiwsky, E. J and Thompson, D. B., 1974: Mathematical models of combination of terrestrial and Satellite Networks. The Canadian Surveyor 28 (1974) 606-615.

Niell, A.E., 1996: Global mapping functions for the atmosphere delay at radio wavelengths. Journal of Geophysical Research, Vol. 101, No. B2, pp 3227-3246.

Sanso, F. (1973): An exact solution of the rototranslation problem. Photogrammetria n 29, pp. 203-216, Elsevier.

Schönemann, P. H., 1996: Generalized solution of the Orthogonal Procrustes Problem, Psychometrika 31 No.1 pp. 1-10.

Zurutuza, J., García, L. and Sevilla, M. J., 2004. Red de estaciones GPS y estación permanente en Gipuzkoa. $4^{\mathrm{a}}$ Asamblea hispanolusa de Geodesia y Geofísica, Figueira da Foz, Portugal. 